\title{
Temporal variations in free core nutation period*
}

\author{
Jianqiao $\mathrm{Xu}^{*}$ and Heping Sun \\ Key Laboratory of Dynamic Geodesy, Institute of Geodesy and Geophysics, \\ Chinese Academy of Sciences, Wuhan 430077, China
}

\begin{abstract}
Based on the nearly diurnal resonance in the tidal gravity observations, the temporal variations in period of the Earth's free core nutation (FCN) are investigated by using the tidal gravity observations of 18-year duration recorded continuously with a superconducting gravimeter (SG) at Brussels. The effects of the global oceanic tide loading and local barometric pressure on the SG observations have been removed by using eleven high-precision global digital models of oceanic tides and barometric pressure measurements recorded simultaneously at the same site. The results indicate that there exist decade-scale variations in the FCN period. The results should be further confirmed by the measurements using other space-based geodetic techniques (such as the very long baseline interferometry) and the SG observations from globally distributed stations.
\end{abstract}

Key words: nearly diurnal resonance; superconducting gravimeter; tidal gravity observations; period of the free core nutation; temporal variation

CLC number: P312.4 Document code: A

\section{Introduction}

The Earth's free core nutation (FCN) is a retrograde free nutational normal mode due to the interaction between the solid mantle and fluid core confined by a deformable solid elliptical core-mantle boundary (CMB). It behaves as the nearly diurnal free wobble (NDFW) with a period of about one day in the terrestrial reference frame. The eigenfrequency of the NDFW, $\sigma_{\text {nd }}$, depends upon ellipticity of the CMB and elasticity in the mantle. As a result, the measurements and investigation of the FCN will provide significant clues and constraint to the studies of the core mantle coupling, the shape of the $\mathrm{CMB}$ in global scale and the distribution of the physical properties near the CMB. An internal pressure, coming from misalign of the instantaneous rotational axis of the elastic solid mantle and the fluid core due to ellipticity of the CMB and exerting on the mantle, will occur and lead to deformation on the CMB and changes in the measurements of Earth's tides (e.g., tides of gravity, tilt, strain) and forced nutations (e.g., very long baseline interferometry, shorten as VLBI) on the Earth's surface. In tidal gravity observations, resonant enhancement will occur in the observations of some diurnal tidal

\footnotetext{
* Received 9 April 2009; accepted in revised form 30 June 2009; published 10 August 2009.

^ Corresponding author. e-mail: xujq@esch.whigg.ac.cn
}

waves with frequencies closed to the eigenfrequency of the NDFW such as $P_{1}, K_{1}, \Psi_{1}$ and $\Phi_{1}$. Based on the resonant pattern in the diurnal tidal gravity observations, the Earth's FCN can be investigated and the corresponding resonance parameters, including the period, quality factor and resonance strength of the FCN, can be retrieved. The results show that the FCN period is about 430 sidereal days seen from inertial space (Neuberg et al, 1987; Florsch et al, 1991; Cummins and Wahr, 1993; Merriam, 1994; Defraigne et al, 1994; Xu et al, 2002; Sato et al, 2004; Herring et al, 1986) and about 30 sidereal days shorter than the one predicted theoretically on the assumption that the Earth is in hydrostatic equilibrium (Wahr, 1981; Mathews et al, 1991a, b). It may attribute to the dynamical ellipticity of the fluid core of the Earth about 5\% larger than one expected for hydrostatic equilibrium (Cummins and Wahr, 1993; Xu et al, 2002). However, previous studies suggested that there was a little difference for the FCN periods retrieved while using the data in different observing periods. Does this difference come from the temporal variations in the FCN period?

From the previous similar studies (Neuberg et al, 1987; Florsch et al, 1991; Cummins and Wahr, 1993; Merriam, 1994; Defraigne et al, 1994; Xu et al, 2002; Sato et al, 2004; Herring et al, 1986), it is found that the period has the highest precision in the retrieved FCN parameters. Long-term accumulation of the measure- 
ments with the superconducting gravimeter (SG) provides a possible potential to study the temporal variations in the FCN period due to its high stability and precision. In this study, the continuous SG observations of 18-year duration at Brussels will be processed comprehensively. The loading effects of local barometric pressure are removed by using station pressure records. The loading effects of the oceanic tides are simulated by using eleven high-precision digital models of global oceanic tides and then removed from the SG observations. The gravimetric parameters will be accurately determined in order to retrieve the FCN parameters. Then, the time changes in the FCN period will be investigated in this study.

\section{SG observations and loading correc- tion of oceanic tides and pressure}

In order to discuss the temporal changes in the FCN period, we separate the SG continuous tidal gravity observations of 18-year duration at Brussels from April 21, 1982 to August 21, 2000 into several segments. All the data have been preprocessed. The length of each data segment with an overlap of a half year is chosen as one and a half years so as to obtain accurately the signatures of tidal waves $\Psi_{1}$ and $\Phi_{1}$. The 36 tidal wave groups, including 20 diurnal, 13 semidiurnal, two terdiurnal and one quarterdiurnal wave groups, are successfully separated, and their gravimetric parameters (i.e., amplitude factor $\delta$ and phase difference $\Delta \varphi$ ) and atmospheric gravity admittance $C$ (i.e., the transform function between the local pressure and gravity) are accurately evaluated by using the software package Eterna (Wenzel, 1996). The HW95, high-precision expansion catalogue of tide-generating potential (Hartmann and Wenzel, 1995), is adopted in the harmonic analysis. The results indicate that the tidal gravity observations from the SG at Brussels are very precise, and the standard deviation is less than $0.2 \times 10^{-8} \mathrm{~m} \cdot \mathrm{s}^{-2}$.

The loading effects of global barometric pressure, which can be simulated theoretically by means of global convolution integration of ground-based pressure measurements and atmospheric gravity Green's functions, are an important noise resource in tidal gravity observations. However, it is found that over $90 \%$ of the loading effects of global pressure come from a region within $50 \mathrm{~km}$ around the station (Merriam, 1992; Niebauer, 1988; Boy et al, 2002), and the correlation coefficient between the pressure variations at the station and its adjacent area is greater than $95 \%$. Therefore, the change pattern of the local pressure can be described as the pressure changes at the station so that it is an ideal way to remove the loading effects of barometric pressure in gravity observations (Kroner and Jentzsch, 1999; Sun and Luo, 1998; Xu et al, 1999). The results indicate that gravity admittance of local pressure $C$ is in the range from $-0.3904 \times 10^{-8}$ $\left(\mathrm{m} \cdot \mathrm{s}^{-2}\right) / \mathrm{hPa}$ to $-0.3160 \times 10^{-8}\left(\mathrm{~m} \cdot \mathrm{s}^{-2}\right) / \mathrm{hPa}$ from 1982 to 2000 at Brussels, and $-0.3487 \times 10^{-8}\left(\mathrm{~m} \cdot \mathrm{s}^{-2}\right) / \mathrm{hPa}$ on average. This result is in good agreement with those from the theoretical simulation and previous similar measurements (Niebauer, 1988; Merriam, 1992, 1993; Crossley et al, 1995; Sun and Luo, 1998; Kroner and Jentzsch, 1999; Xu et al, 1999, 2004; Boy et al, 2002).

Only the diurnal tidal gravity observations are related to the FCN resonance and concerned in this study. Thanks to its high ratio of signal to noise and less influence from surrounding environment disturbance, tidal wave $\mathrm{O}_{1}$ is regarded as an example to analyze the temporal variations in the gravimetric amplitude factors of the diurnal tides observed with the SG at Brussels. Figure 1 depicts time changes in the amplitude factor $\delta$ of $\mathrm{O}_{1}$ from 1982 to 2000 . Meanwhile, those for the tidal wave $\mathrm{M}_{2}$ are also described in Figure 1 for comparison. From Figure 1, it is found that the amplitude factor of $\mathrm{O}_{1}$ locates in the range of 1.15251 and 1.15525 , and is about 1.15333 on average. The magnitude of its variations is less than $\pm 0.17 \%$ and slightly greater than the one for the amplitude factor of $\mathrm{M}_{2}$. It implies that the SG measurements are very stable. However, it is found that the changes in the amplitude factors of $\mathrm{O}_{1}$ and $\mathrm{M}_{2}$ correlate strongly to each other, and the correlation coefficient is almost as large as $100 \%$ before 1990 . On the contrary, they do not correlate at all after 1990. It suggests that there probably exist the scale problems in the

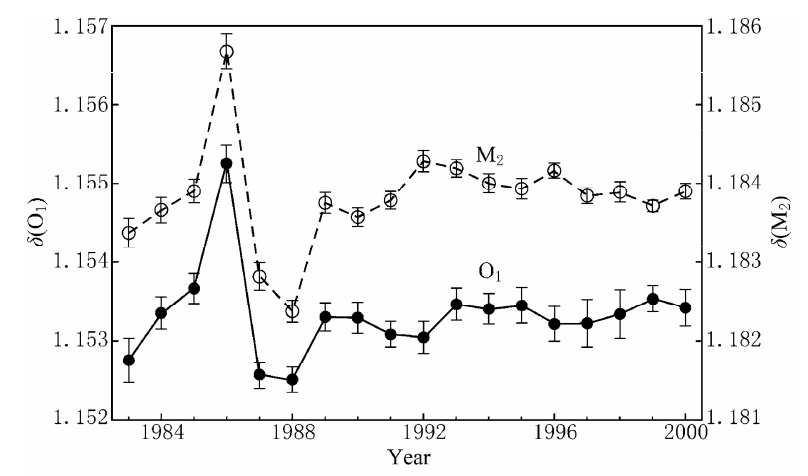

Figure 1 Temporal variation in the gravimetric amplitude factors $\delta$ of tidal waves $\mathrm{O}_{1}$ and $\mathrm{M}_{2}$. 
observations before 1990. Therefore, in order to weaken the effects of calibration discrepancy on the final results, the determined gravimetric amplitude factors of $\mathrm{O}_{1}$ and $\mathrm{M}_{2}$ are scaled to reference values provided in the global experimental model for gravity tides (Xu et al, 2004), in which the tidal gravity observations from a worldwide SG network, the loading effects of global oceanic tides, the nearly diurnal resonance and the latitude dependence of amplitude factors are taken into account. The resulted gravimetric parameters in diurnal frequency band are much closed to those provided in the theoretical model developed by Mathews (2001), while the gravimetric parameters in semidiurnal frequency band are more closed to those developed by Dehant et al (1999).

The loading effects of global oceanic tides are the predominant noise in the observations of gravity tides. The loading correction vector $\boldsymbol{L}(L, \lambda)$ to a certain gravity tidal wave can be deduced by means of global convolution integration of the gravity Green's functions under surface loads and the height of the oceanic tide wave (Xu and Mao, 1988; Agnew, 1997), and then removed from the tidal gravity observations. In this study, eleven high-precision models of global oceanic tides, including Sch80 (Schwiderski, 1980), Csr3.0 (Eanes and Bettadpur, 1995), Csr4.0 (Eanes and Schuler, 1999), Fes952, Fes99, Fes02 (Le Provost et al, 1994; Lefèvre et al, 2002), Tpxo02 (Egbert et al, 1994), Ori96 (Matsumoto et al, 1995), Ag95 (Andersen, 1995), Nao99 (Matsumoto et al, 2000) and Got00 (Ray, 1999), are used to remove accurately the oceanic tide loading from the SG tidal gravity observations. These oceanic tide models have been adopted widely in the ground- and space-based geodetic techniques. Figure 2 depicts the amplitudes of observed residuals (phasor difference of the observed vector and its theoretical prediction of a certain signature) of tidal waves $\mathrm{O}_{1}$ and $\mathrm{M}_{2}$ measured with the $\mathrm{SG}$ at Brussels before and after removal of loading effects of the oceanic tides. It is well known that Brussels locates on the central Europe, where the loading effects of global oceanic tides on tidal gravity observations mainly come from the contribution of the oceanic tides in the Atlantic ocean. Their effects on the gravity tides in the semidiurnal band are much more significant than those in the diurnal band. In consideration of the tidal gravity observations with the SG, it is found that there is some uncertainty in oceanic tide models and no model is decisively better than the others for loading correction to tidal gravity observations by using different oceanic tide models (Baker and Bos, 2001; Boy et al, 2003; Xu et al,
2004; Sun et al, 2005; Ducarme et al, 2007). It is suggested that a mean tidal loading vector based on plural oceanic tide models should give the most stable solutions for the tidal gravity observations, especially for the study of liquid core resonance (Xu et al, 2004; Sun et al, 2005; Ducarme et al, 2007). In this study, the mean loading vector based on all eleven models is used as the final loading correction vector for a certain tidal component in order to depress effectively uncertainty in an individual oceanic tide model.

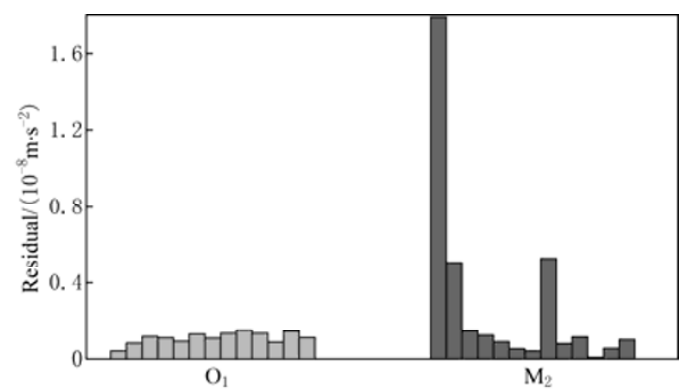

Figure 2 Residual amplitude of waves $\mathrm{O}_{1}$ and $\mathrm{M}_{2}$ after removal of oceanic tide loading. Each group of histograms represent the residual amplitude for no oceanic tide correction and removal of the oceanic tide loading by using the oceanic tide models Sch80, Csr3.0, Csr4.0, Fes952, Fes99, Tpxo02, Fes02, Ori96, Ag95, Nao99, Got00 and their mean, respectively, in turn from left to right.

Most of the oceanic tide models only involve the co-tidal maps of the main tidal components including four diurnal and four semidiurnal waves (i.e., $\mathrm{Q}_{1}, \mathrm{O}_{1}, \mathrm{P}_{1}$, $\mathrm{S}_{1}, \mathrm{~N}_{2}, \mathrm{M}_{2}, \mathrm{~S}_{2}$ and $\mathrm{K}_{2}$ ). The loading vectors of other weaker components, especially those of the diurnal tidal waves with frequency near the NDFW eigenfrequency (e.g., $\Phi_{1}$ and $\Psi_{2}$ ), should be estimated by means of interpolation or extrapolation in frequency domain according to the simulated loading vectors of these eight tidal components ( $\mathrm{Xu}$ et al, 2004; Sun et al, 2005). In the procedure of interpolation or extrapolation, the heights of equilibrium tides and the nearly diurnal resonant pattern of oceanic tides (Wahr and Sasao, 1981) are taken into account.

\section{Estimation of the FCN resonance parameters}

The existence of the FCN results in the nearly diurnal resonance in tidal gravity observations. The gravimetric parameters of a certain diurnal tidal component with frequency $\sigma$ close to the NDFW eigen-frequency $\widetilde{\sigma}_{\text {nd }}$ (e.g., $\mathrm{P}_{1}, \mathrm{~K}_{1}, \Psi_{1}$ and $\Phi_{1}$ ) should be resonantly en- 
hanced, and can be described as (Xu et al, 2002; Hinderer et al, 1991)

$$
\delta(\sigma) \mathrm{e}^{\mathrm{i} \Delta \varphi(\sigma)}=\delta_{0} \mathrm{e}^{\mathrm{i} \Delta \varphi_{0}}+\frac{\widetilde{a}}{\sigma-\widetilde{\sigma}_{\text {nd }}},
$$

where $\delta_{0}$ and $\Delta \varphi_{0}$ are frequency-independent components in the gravimetric parameters of the diurnal tides and not contaminated by the nearly diurnal resonance, $\widetilde{a}=a_{\mathrm{r}}+\mathrm{i} a_{\mathrm{i}}$ the complex resonance strength and $\widetilde{\sigma}_{\text {nd }}=$ $\sigma_{\mathrm{r}}+\mathrm{i} \sigma_{\mathrm{i}}$ the complex eigen-frequency of the NDFW. The quality factor of the NDFW can be expressed as $Q=$ $\sigma_{\mathrm{r}} /\left(2 \sigma_{\mathrm{i}}\right)$. In tidal gravity observations, the tidal wave $\mathrm{O}_{1}$ is regarded as a reference due to its high ratio of signal to noise and less influence from surrounding environment disturbances. Meanwhile, its observations are little influenced by the FCN resonance due to its frequency far away from the NDFW eigen-frequency. The corresponding signatures of $\mathrm{O}_{1}$ are removed from equation (1) in order to depress the influences of the local background perturbations on the simulation of the FCN parameters, and we obtain

$$
\begin{gathered}
\delta(\sigma) \mathrm{e}^{\mathrm{i} \Delta \varphi(\sigma)}-\delta\left(\mathrm{O}_{1}\right) \mathrm{e}^{\mathrm{i} \Delta \varphi\left(\mathrm{O}_{1}\right)}= \\
\frac{\tilde{a}}{\sigma-\widetilde{\sigma}_{\text {nd }}}-\frac{\tilde{a}}{\sigma\left(\mathrm{O}_{1}\right)-\widetilde{\sigma}_{\text {nd }}} .
\end{gathered}
$$

Based on equation (2) or its reformation, the FCN resonance parameters can be retrieved by using the tidal gravity observations from a certain station or plural stations (Neuberg et al, 1987; Florsch et al, 1991; Cummins and Wahr, 1993; Merriam, 1994; Defraigne et al, 1994; Xu et al, 2002; Sato et al, 2004). As well known, the SG used in tidal gravity measurements is a relative gravimeter and should be calibrated in order to determine both the scale factor and response lag. From equation (2), it is easily found that the calibration error involved in the tidal gravity observations would significantly lead to systematical discrepancy in estimation of the FCN parameters. In order to depress the effects of calibration error, the left-hand and right-hand sides of equation (2) are divided by the observed and theoretical gravimetric parameters of $\mathrm{O}_{1}$, respectively. Retaining the predominant component, we have

$$
\begin{aligned}
& \frac{\delta(\sigma)}{\delta\left(\mathrm{O}_{1}\right)} \mathrm{e}^{\mathrm{i}\left[\Delta \varphi(\sigma)-\Delta \varphi\left(\mathrm{O}_{1}\right)\right]}-1= \\
& \frac{\widetilde{s}}{\sigma-\widetilde{\sigma}_{\text {nd }}}-\frac{\widetilde{s}}{\sigma\left(\mathrm{O}_{1}\right)-\widetilde{\sigma}_{\text {nd }}},
\end{aligned}
$$

with $\widetilde{s}=\widetilde{a} / \delta_{0} \mathrm{e}^{\mathrm{i} \Delta \varphi_{0}}=s_{\mathrm{r}}+\mathrm{i} s_{\mathrm{i}}$, called still the resonance strength. From equation (3), it is found that the ratio of the amplitude factors in the left-hand side may significantly depress the effects of the discrepancy in calibration of the scale factor, while the difference of the phase differences should reduce the effects of the discrepancy in calibration of the response lag.

From the previous studies, it is found that the quality factor of the FCN, $Q$, is sometimes retrieved as a physically impossible negative value because the (absolute) value of $\sigma_{\mathrm{i}}$ is extremely small and usually in the same order of the estimated error. Following the suggestion by Florsch and Hinderer (2000), prior information for $Q$, a non-negative function, is introduced into the computation model in order to avoid this problem, it is given as

$$
Q=\frac{\sigma_{\mathrm{r}}}{2 \sigma_{\mathrm{i}}}=10^{x} .
$$

Substituting equation (4) into equation (3), the computation model for the FCN parameters is improved as

$$
\begin{gathered}
\frac{\delta(\sigma)}{\delta\left(\mathrm{O}_{1}\right)} \mathrm{e}^{\mathrm{i}\left[\Delta \varphi(\sigma)-\Delta \varphi\left(\mathrm{O}_{1}\right)\right]}-1= \\
\frac{s_{\mathrm{r}}+\mathrm{i} s_{\mathrm{i}}}{\sigma-\sigma_{\mathrm{r}}\left(1+0.5 \mathrm{i} \cdot 10^{-x}\right)} \\
-\frac{s_{\mathrm{r}}+\mathrm{i}_{\mathrm{i}}}{\sigma\left(\mathrm{O}_{1}\right)-\sigma_{\mathrm{r}}\left(1+0.5 \mathrm{i} \cdot 10^{-x}\right)}
\end{gathered}
$$

Based on equation (5), the FCN parameters, including its period, can be retrieved by using the SG tidal gravity observations. In order to estimate well the FCN period, the $Q$ value is constrained to be no greater than $10^{6}$.

In order to discuss the influences of uncertainty in the oceanic tide models on evaluation of the FCN, the tidal gravity observations with the SG in 1983 are chosen as an example. Based on the eleven oceanic tide models, the oceanic tide loading are removed and then the FCN parameters are retrieved, respectively. The numerical results indicate that the different oceanic tide models significantly result in differences of several sidereal days in the retrieved FCN period. While the mean loading vector based on all eleven models is used as the final loading correction vector for each tidal component; the FCN period is retrieved as $(432.2 \pm 41.5)$ sidereal days closed to the results obtained in the previous studies (Neuberg et al, 1987; Florsch et al, 1991; Cummins and Wahr, 1993; Merriam, 1994; Defraigne et al, 1994; $\mathrm{Xu}$ et al, 2002; Sato et al, 2004; Herring et al, 1986). It implies that mean loading vectors of plural oceanic tide models can significantly depress uncertainty in those from an individual model and lead to more acceptable 
estimation of the FCN period.

The FCN parameters are retrieved for each data segment of one and a half years duration with an overlap of a half year, and then the temporal variations in the FCN period are obtained and presented in Figure 3 based on the 18-year SG measurements at Brussels. It is found that there is significant difference in the evaluated FCN period for different observing intervals. It is in the range of 414.5-478.8 sidereal days, and 442.5 sidereal days on average. In general, the FCN period retrieved in this study is significantly different from the one evaluated by stacking the observations from globally distributed stations due to the influences of local environment perturbations, uncertainties in the oceanic tide models and possibly systematical discrepancy in measurements of an individual instrument. However, because these effects always occur during the whole measurements, they should not distort heavily the long-term trends of time changes in the FCN period. From the numerical results shown in Figure 3, it is found that there probably is a long-term trend of about decade-scale variations in the FCN period. In the consideration of the relatively large standard deviation of the FCN period based on tidal gravity observations with an individual SG, the results should be confirmed by the other space-based geodetic techniques (such as VLBI) and the SG observations from globally distributed stations.

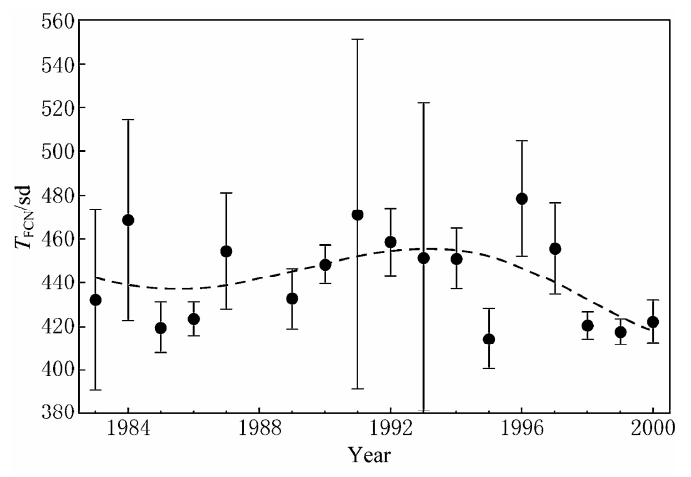

Figure 3 Temporal variations in the $\mathrm{FCN}$ period, $T_{\mathrm{FCN}}$ in unit of sidereal day, retrieved from the SG observations at Brussels.

The previous studies indicated that the FCN period is strongly dependent on the dynamic ellipticity of the core and elasticity in the mantle (Mathews et al, 1991a, b). In addition, the environment perturbations would be involved in the tidal gravity observations and then also affect the retrieved FCN period. However, in the most significant perturbations, including the variations in local and global barometric pressure and hydrological movements, and their associated geodynamical phenomena, no decade variations have been found. Therefore, the decade variations in the FCN period should be related to dynamical behaviors in the Earth's interior. It is found that there also exist the decade variations in the length of day (LOD, i.e., Earth's rotational rate) which should come from the contribution of the electromagnetic coupling between the core and the mantle (Pais and Hulot, 2000). This fact may provide a possible clue for the mechanism of the FCN period variations in the same scale. On the one hand, the electromagnetic coupling between the core and the mantle will lead to changes of several days in the FCN period. On the other hand, there are obviously decade variations in the LOD related to the electromagnetic coupling between the core and mantle. The LOD decade variations would result in the corresponding changes in the dynamic ellipticity of the whole Earth and its core. In a word, electromagnetic coupling at the core-mantle boundary probably is one of the sources of the decade variations in the FCN period. Of course, the relatively large standard deviation of the FCN period retrieved from the observations of an individual SG does not allow us to discuss further the mechanics of temporal variations in the FCN period so far.

\section{Conclusions}

After effective loading correction of the local atmospheric pressure and oceanic tides, the FCN parameters, especially the FCN period, can be well retrieved by using the tidal gravity observations with the $\mathrm{SG}$ at an individual station. There are some uncertainties in the oceanic tide models, which cause the difference of several days in the estimated FCN period. However, while the mean loading vector based on all eleven models is used as the final loading correction vector for each tidal component, the effects of uncertainty on the evaluated FCN period can be depressed significantly and result in more acceptable estimation. The computation model for the FCN parameters is improved in order to depress the effects of local environment background noise and calibration errors of the instrument upon the retrieval of the FCN parameters. However, it is found that the resulted FCN period from the SG tidal gravity observations at individual Brussels is slightly different from the one by stacking the observations from globally distributed stations due to the influences of local environment perturbations, uncertainties in the oceanic tide models and possibly existed systematical discrepancy in measure- 
ments of an individual instrument. There exist decadescale variations in the FCN period. Of course, the results should be confirmed by other space-based geodetic techniques (such as the VLBI) and the SG observations from globally distributed stations. The mechanism of temporal variations in the FCN period should be investigated further.

Acknowledgements This study is supported by the Key Project of the Knowledge Innovation of Chinese Academy of Sciences (Grant No. KZCX2-YW-133, KZCX2-YW-Q08-2) and National Natural Science Foundation of China (Grant No. 40874038 and 40730316).

\section{References}

Agnew D C (1997). NLOADF: A program for computing ocean tide loading. $J$ Geophys Res 102(B3): 50 109-51 110.

Andersen O B (1995). Global ocean tides from ERS-1 and TOPEX/POSEIDON altimetry. $J$ Geophys Res 100: 25 249-25 259.

Baker T F and Bos M S (2003). Validating Earth and ocean models using tidal gravity measurements. Geophys J Int 152: 468-485.

Boy J P, Gegout P and Hinderer J (2002). Reduction of surface gravity from global atmospheric pressure loading. Geophys J Int 149: 534-545.

Boy J P, Llubes M, Hinderer J and Florsch N (2003). A comparison of tidal ocean loading models using superconducting gravimeter data. $J$ Geophys Res 108(B4): 2 193, doi:10.1029/2002JB002050.

Crossley D, Jensen O G and Hinderer J (1995). Effective barometric admittance and gravity residuals. Phys Earth Planet Inter 90: 221-241.

Cummins P R and Wahr J M (1993). A study of the Earth's free core nutation using international deployment of accelerometers gravity data. $J$ Geophys Res 98(B2): 2 091-2 103.

Defraigne P, Dehant V and Hinderer J (1994). Staking gravity tide measurements and nutation observations in order to determine the complex eigenfrequency of nearly diurnal free wobble. $J$ Geophys Res 99(B5): 9 2039213.

Dehant V, Defraigne P and Wahr J (1999). Tides for a convective Earth. $J$ Geophys Res 104: 1 035-1 058.

Ducarme B, Sun H P and Xu J Q (2007). Determination of the free core nutation period from tidal gravity observations of the GGP superconducting gravimeter network. J Geod 81: 179-187.

Eanes R J and Bettadpur S V (1995). The CSR 3.0 Global Ocean Tide Model: Diurnal and Semi-diurnal Ocean Tides From TOPEX/POSEIDON Altimetry. CSR Technical Memorandum 95-06, Center for Space Research, The University of Texas at Austin, Austin, Texas, USA, 1-25.

Eanes R J and Schuler A (1999). An improved global ocean tide model from TOPEX/Poseidon altimetry: CSR4.0. EGS, 24th General Assembly. The Hague, Netherlands, April 19-23.

Egbert G, Bennett A and Foreman M (1994). TOPEX/Poseidon tides estimated using a global inverse model. J Geophys Res 99(C12): 24 821-24 852.

Florsch N, Chambat F, Hinderer J and Legros H (1991). A simple method to retrieve the complex eigenfrequency of the Earth's nearly diurnal-free wobble: Application to the Strasbourg superconducting gravimeter data. Geophys $J$ Int 116: 53-63.

Florsch $\mathrm{N}$ and Hinderer $\mathrm{J}$ (2000). Bayesian estimation of the free core nutation parameters from the analysis of precise tidal gravity data. Phys Earth Planet Inter 117: 21-35.

Hartmann T and Wenzel G (1995). The HW95 tidal potential catalogue. Geophys Res Lett 22(24): 3 553-3 556.

Herring T A, Gwinn C R and Shapiro I I (1986). Geodesy by radio interferometry: Studies of the forced nutations of the earth. I-Data analysis. $J$ Geophys Res 91(B5): 4 745-4 754.

Hinderer J, Legros H and Crossley D (1991). Global Earth dynamics and induced gravity changes. $J$ Geophys Res 96: 20 257-20 265 .

Kroner C and Jentzsch G (1999). Comparison of different barometric pressure reductions for gravity data and resulting consequences. Phys Earth Planet Inter 115: 205-218.

Le Provost C, Genco M L, Lyard F, Vincent P and Canceil P (1994). Tidal spectroscopy of the ocean tides from a finite element hydrodynamic model. $J$ Geophys Res 99(C12): 24 777-24 798.

Lefèvre F, Lyard F H, Le Provost C and Schrama E J O (2002). FES99: A global tide finite element solution assimilating tide gauge and altimetric information. J Atmo Oceanic Technol 19: 1345-1 356.

Mathews P M (2001). Love numbers and gravimetric factor for diurnal tides. $J$ Geod Soc Japn 46(4): 231-236.

Mathews P M, Buffett B A, Herring T A and Shapiro I I (1991a). Forced nutations of the Earth: Influence of inner core dynamics 1. Theory. J Geophys Res 96: 8 219-8 242.

Mathews P M, Buffett B A, Herring T A and Shapiro I I (1991b). Forced nutations of the Earth: Influence of inner core dynamics 2. Numerical results and comparison. J Geophys Res 96: 8 243-8 257.

Matsumoto K, Ooe M, Sato T and Segawa J (1995). Ocean tide model obtained from TOPEX/POSEIDON altimetry data. $J$ Geophys Res 100(C12): $25319-25330$.

Matsumoto K, Takanezawa T and Ooe M (2000). Ocean tide models developed by assimilating TOPEX/POSEIDON altimeter data into hydrodynamical model: A global model and a regional model around Japan. J Oceanography 56: 567-581.

Merriam J B (1992). Atmospheric pressure and gravity. Geophys J Int 109: $488-500$.

Merriam J B (1993). The atmospheric pressure correction in gravity at Cantley Quebec. In: Hsu H-T ed. Proc 12th Int Sympos on Earth Tides. Science Press, Beijing, 161-168.

Merriam J B (1994). The nearly diurnal free wobble resonance in gravity measured at Cantley, Quebec. Geophys J Int 119: 369-380.

Neuberg J, Hinderer J and Zürn W (1987). Stacking gravity tide observations in central Europe for the retrieval of the complex eigenfrequency of the nearly diurnal free wobble. Geophys $J$ R astr Soc 91: 853-868.

Niebauer T M (1988). Correcting gravity measurements for the effects of local air pressure. J Geophys Res 93(B7): 7 989-7 991.

Pais A and Hulot G (2000). Length of day decade variations, torsional oscillations and inner core superrotation: Evidence from recovered core surface zonal flows. Phys Earth Planet Inter 118(3-4): 291-316.

Ray R D (1999). A Global Ocean Tide Model From TOPEX/POSEIDON Altimetry: GOT99.2. NASA Technical Memorandum 209478, Goddard Space Flight Center, 1-58.

Sato T, Tamura Y, Matsumoto K, Imanishi Y and McQueen H (2004). Parameters of the fluid core resonance inferred from superconducting gravimeter data. J Geodyn 38: 375-389.

Schwiderski E W (1980). Ocean tides I: Global ocean tidal equations. Mar Geod 3: 161-217.

Sun H P, Ducarme B, Xu H Z, Vandercoilden L, Xu J Q and Zhou J C (2005). Adaptability of the ocean and Earth tidal models based on global observations of the superconducting gravimeters. Science in China (Series D) 48(11): 1 859- 1869.

Sun H P and Luo S C (1998). Theoretical computation and detection of the atmospheric gravity signal. Chinese J Geophys 41(5): 634-641.

Wahr J M (1981). Body tides on an elliptical, rotating, elastic and oceanless earth. Geophys J R astr Soc 64: 677-703.

Wahr J M and Sasao T (1981). A diurnal resonance in the ocean tide and in the earth's load response due to the resonant free 'core nutation'. Geophys $J R$ astr Soc 64: 747-765.

Wenzel G (1996). The nanogal software: Data processing package ETERNA 3.3. Bull Inf Marées Terrestres 124: 9 425-9 439.

Xu H Z and Mao W J (1988). Model for ocean tide loading correction of China mainland. Science in China (Series B) 9: 984-994 (in Chinese).

Xu J Q, Hao X H and Sun H P (1999). Influence of atmospheric pressure on tidal gravity at Wuhan station. Acta Geodaetica et Cartographica Sinica 28(1): 75-79 (in Chinese with English Abstract).

Xu J Q, Sun H P and Ducarme B (2004). A global experimental model for gravity tides of the Earth. J Geodyn 38(3-5): 291-304.

Xu J Q, Sun H P and Luo S C (2002). Study of the Earth's free core nutation by tidal gravity data recorded with international superconducting gravimeters. Science in China (Series D) 45(4): 337-347. 